
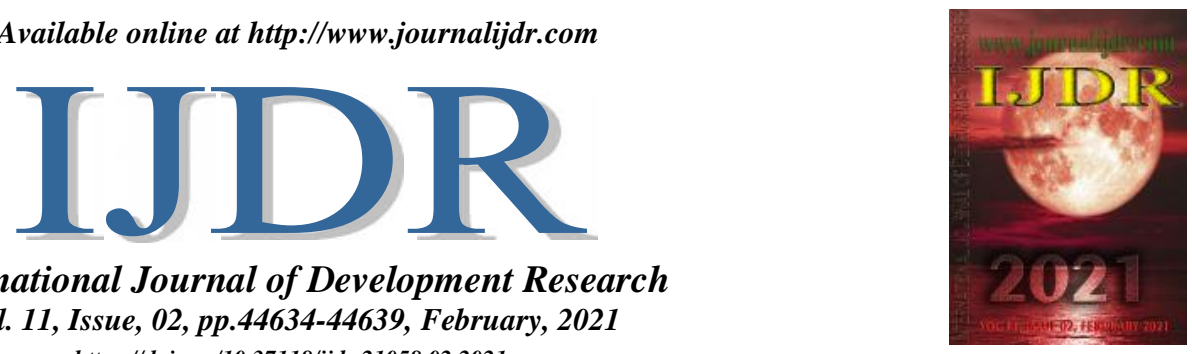

ISSN: 2230-9926

International Journal of Development Research

Vol. 11, Issue, 02, pp.44634-44639, February, 2021

https://doi.org/10.37118/ijdr.21058.02.2021

\title{
HANSENÍASE: REVISÃO SISTEMÁTICA DA LITERATURA SOBRE 0 ESTIGMA VIVENCIADO POR SEUS PORTADORES
}

\author{
Mariane Costa Santos de Tavares ${ }^{1}$, Suzana Cristina Silva Ribeiro², Carolina Palmeira Teixeira \\ Martins $^{2}$, Ivan Gilson Silva Moura ${ }^{3}$, Lorena Vieira Fernandez de Araújo ${ }^{4}$, Cintia Maria Koenig ${ }^{5}$, \\ Jennifer Rodrigues Correia ${ }^{6}$, Amanda Rocha Vasconcelos ${ }^{6}$, Larissa Feli de Sousa Oliveira ${ }^{6}$, \\ Ana Virgínia Figueira Dubois Mendes ${ }^{6}$, Eduardo Fernandes da Silva ${ }^{6}$, Karine Thamires Costa \\ Nascimento $^{7}$ and Karina Pereira Neris ${ }^{8}$
}

1 Autor Correspondente, Fisioterapeuta, Pós-Graduada em Terapia Intensiva, Discente do Curso de Graduação em Medicina. Faculdades Santo Agostinho - FASA, Vitória da Conquista - Bahia, ${ }^{2}$ Docente do Curso de Graduação em Medicina. Faculdades Santo Agostinho - FASA, Vitória da Conquista - Bahia; ${ }^{3}$ Médico. Psiquiatra, Docente do Curso de Graduação em Medicina, Faculdades Santo Agostinho - FASA, Vitória da Conquista - Bahia

${ }^{4}$ Fisioterapeuta, Especialista em Fisioterapia Uroginecológica pelo CBES-SP, Mestre em Ciências Ambientais pela UFOB BA, Discente do Curso de Graduação em Medicina. Faculdades Santo Agostinho - FASA, Vitória da Conquista - Bahia; 5 Médica, Especialista em Saúde Coletiva pela Universidade Federal da Bahia - UFBA, Graduação em enfermagem, PósGraduada em Urgência e Emergência, Pós-Graduada em enfermagem em estética pela FAVENI; ${ }^{6}$ Discente do Curso de Graduação em Medicina. Faculdades Santo Agostinho - FASA, Vitória da Conquista - Bahia; ${ }^{7}$ Discente do Curso de Graduação em Medicina. Universidade Estadual da Bahia - UESB, Vitória da Conquista - Bahia; 8 Discente do Curso de Graduação em Odontologia, Uninassau, Vitória da Conquista - BA

\section{ARTICLE INFO}

\section{Article History:}

Received $07^{\text {th }}$ December, 2020

Received in revised form

$09^{\text {th }}$ December, 2020

Accepted $24^{\text {th }}$ January, 2021

Published online $24^{\text {th }}$ February, 2021

Key Words:

Leprosy,

Neglected diseases,

Social Stigma.

*Corresponding author:

Mariane Costa Santos de Tavares

\begin{abstract}
Objective: To analyze the existence of correlation between the diagnosis of Leprosy and the experience of stigma suffered/perceived by the patient of the disease. Methods: Systematic literature review study, structured as proposed in the PRISMA (Preferred Reporting Items for Systematic Reviews and Meta-Analyses) methodology, and the literary search was performed in the databases linked to the Medical Literature Analysis and Retrieval System Online (MEDLINE), and Latin American and Caribbean Literature on Health Sciences (LILACS), using the descriptors in health sciences (DECS): Leprosy, Social Stigma and Prejudice. Results: eleven studies were selected for data analysis and discussion, according to identified search and selection criteria. Final considerations: The suspected and/or conformed diagnosis of leprosy brings to the individual great psychic suffering, either due to the unknowledge about the treatment and concept of the disease, as well as the stigma that still exists about the disease. Exclusion, prejudice, and social isolation of affected individuals and their families were excluded.
\end{abstract}

Copyright @ 2021, Geovana Pires Araujo Lima et al. This is an open access article distributed under the Creative Commons Attribution License, which permits unrestricted use, distribution, and reproduction in any medium, provided the original work is properly cited.

Citation: Geovana Pires Araujo Lima, Daniel Vianna GoesAraujo, Josiane Dantas Viana Barbosa, Valter Estevão Beall and Juliano ZaffalonGerber. "Hanseníase: revisão sistemática da literatura sobre o estigma vivenciado por seus portadores", International Journal of Development Research, 11, (02), 44634-44639.

\section{INTRODUCTION}

A hanseníase é uma patologia infectocontagiosa crônica, que compromete a pele e nervos periféricos (trigêmeo, facial, tibial posterior, ulnar, mediano, radial e fibular comum) podendo trazer graves consequências aos pacientes quando não há uma intervenção precoce .
Trata-se de uma doença causada pelo bacilo de Hansen (Mycobateriumleprae), identificado inicialmente em 1873 pelo bacteriologista norueguês Gerhard HenrikArmauer Hansen. A hanseníase, referida historicamente como "lepra", teve seu nome alterado no contexto brasileiro, visando sobretudo, a redução do estigma relacionado à doença enfrentado pelos seus portadores (Araújo, 2019). 
No Brasil, entre os anos de 2014 e 2018 foram diagnosticados 140.578 novos casos de hanseníase, sendo que 55,2\% destes casos ocorreram em indivíduos do sexo masculino. A faixa etária de maior incidência de novos casos foi de 50-59 anos, totalizando 26245 indivíduos (Brasil, 2020). Estima-se que nas regiões Norte, Nordeste e Centro-Oeste brasileiras surjam em média 47 mil novos casos por ano. O Brasil é considerado o segundo país com maior incidência de Hanseníase, perdendo apenas para a Índia (Araújo, 2016). Segundo o Boletim Epidemiológico brasileiro o Nordeste possui o maior número de casos de hanseníase entre indivíduos analfabetos $(11,8 \%)$. Outro dado relevante é que, embora se observe redução do número de casos em todas as regiões do país, verifica-se flutuação desse indicador nas regiões Norte, Centro-Oeste e Nordeste. Ainda com tal flutuação, o documento aponta que em análise anterior as regiões Norte e Nordeste eram consideradas "precárias" em relação à proporção dos contatos examinados e busca ativa por novos casos. $\mathrm{Na}$ análise atual, verifica-se o avanço de tais regiões para o nível regular, o que significa que a proporção de contatos examinados ocorre em 74,5 a $81,4 \%$ dos casos (Brasil, 2020). A literatura aponta que os pacientes com hanseníase sofrem negligência do poder público e serviços de saúde no diagnóstico precoce, acompanhamento dos casos, bem como orientações qualificadas. Dentre estes há uma parcela considerável que realizam o tratamento de maneira irregular ou abandonam o mesmo, elevando ainda mais o impacto da doença. Grande parte dos doentes diagnosticados, por sua vez, esconde a patologia com receio do preconceito e da exclusão. Conforme relatado pelos pesquisadores, embora a Hanseníase hoje tenha cura, ainda é uma patologia bastante estigmatizada (Sousa, 2013). A hanseníase é historicamente descrita como uma doença estigmatizada pela sociedade. Como uma doença milenar, muito se atribuía a ocorrência da "lepra" com castigo divino, sendo seus portadores considerados impuros, e não dignos de cuidados ou atenção ((Pinheiro, et al., 2018). A doença, sobretudo em estágios avançados, promove úlceras na pele, amputações e deformidades corporais, o que eleva a exclusão social e preconceito contra seus portadores (Lopes, et al., 2020). O objetivo do presente estudo foi realizar analisar a existência de correlação entre o diagnóstico de Hanseníase e a vivência de estigma sofrido/percebido pelo portador da doença.

\section{MÉTODOS}

Trata-se de uma revisão sistemática de literatura. O estudo foi estruturado conforme proposto na metodologia PRISMA (PreferredReportingItems for Systematic Reviews and MetaAnalyses), visando maior qualidade na busca literária e sistematização dos resultados encontrados. A declaração PRISMA utilizada neste estudo consiste em uma lista de verificação composta por vinte e sete itens, e a utilização de um diagrama de fluxo de quadro fases, priorizando clareza e transparência na execução da revisão sistemática (Liberati, 2009). A busca por estudos se deu nas bases de dados vinculadas à Medical LiteratureAnalysis and Retrieval System Online (MEDLINE) e Literatura Latino-americana e do Caribe em Ciências da Saúde (LILACS), utilizando-se os descritores em ciências da saúde (DECS): Hanseníase, Estigma social e Preconceito. A busca foi realizada de forma pareada, utilizando-se o booleano AND para a associação dos termos, sendo estruturados como descritores pareados: "hanseníase AND estigma social", "Hanseníase AND Preconceito" e seus equivalentes nos idiomas inglês ("leprosy AND social stigma", Leprosy AND Prejudice) e espanhol ("lepra AND estigma social", "Lepra AND prejuicio"). Foram considerados somente artigos originais sobre hanseníase, enfatizando o estigma/preconceito sofrido e/ou percebido, disponíveis na íntegra nos idiomas inglês, português e espanhol, publicados entre os anos de 2016 a 2021. Foram excluídos estudos que não correlacionassem o diagnóstico de hanseníase com o estigma social e/ou preconceito, bem como estudos de revisão literária, ou que não especificavam adequadamente as estratégias metodológicas adotadas. Os critérios de elegibilidade dos estudos ocorreram por meio dos critérios de SPICE e estão detalhados no Quadro 1. A pergunta norteadora do estudo foi estruturada com base na estrutura SPICE, considerando o cenário, perspectiva, intervenção/ Fenômeno de interesse, comparação e avaliação. Considerou-se cenário o diagnóstico confirmado de hanseníase, a perspectiva a autopercepção do estigma, e o fenômeno de interesse a exclusão, estigma e/ou preconceito. Buscou-se comparar ainda a relação entre estigma sofrido/e ou percebido em diferentes contextos socioeconômicos, étnicos, e níveis de escolaridade. Após a definição dos eixos SPICE a pergunta norteadora do estudo estabelecida foi: Existe correlação entre o diagnóstico de Hanseníase e a vivência de estigma sofrido/percebido pelo portador da doença ?. Sendo ainda considerada a segunda questão: a percepção de estigma/preconceito possui relação com o nível socioeconômico, grau de escolaridade ou aspectos étnicos?. A busca foi feita por dois pesquisadores independentes, visando maior acurácia na seleção dos estudos. Posteriormente, somou-se os estudos selecionados pelos dois pesquisadores e realizou-se a análise dos resumos dos estudos, considerando os critérios de inclusão e exclusão propostos, e caso a leitura do resumo não fosse suficiente para definição da adequabilidade procedia-se a leitura na íntegra do estudo. Foram incluídos estudos validados pelos dois pesquisadores. Em situações que houvesse ausência de consenso sobre a adequabilidade do estudo poderia ser solicitado um terceiro parecer de outro autor, entretanto, não foi necessário tal recurso. Por fim, na terceira etapa os estudos selecionados foram lidos integralmente, e procedeu-se a extração dos dados necessários para atingir os objetivos da pesquisa. Visando melhor acurácia e organização dos dados elaborou-se uma planilha descritiva utilizandose o Microsoft Excel ${ }^{\circledR} 2013$, com as informações: autores, ano, local da publicação, título, tipo de estudo, metodologia e resultados. Foi utilizada ainda a ferramenta da Cochrane de avaliação de risco de viés (Cochrane Risk of Bias Tool), buscando melhorar a qualidade e adequabilidade dos estudos selecionados (Carvalho et al., 2013). A análise dos estudos se deu através da abordagem qualitativa dos pontos apresentados na estratégia SPICE, buscando avaliar a temática estudada, o estigma sofrido e/ou percebido, bem como a relação entre tal estigma, e os contextos étnicos, socioeconômicos, e níveis de escolaridade dos sujeitos analisados.

\section{RESULTADOS}

A busca e seleção dos estudos obedeceu aos critérios acima estabelecidos, sendo que a análise de adequabilidade destes seguiu a seleção dos títulos, posterior análise dos resumos, e por fim, leitura completa dos estudos selecionados, e análise qualitativa dos dados apresentados. No Fluxograma 1 está apresentado o processo de seleção dos estudos, conforme proposto pela metodologia PRISMA adotada neste estudo (Fluxograma 1). Com o intuito de abordar os critérios estabelecidos estudados cada um dos estudos selecionados foi analisado quanto ao diagnóstico de hanseníase, percepção frente ao diagnóstico, estigma sofrido e/ou percebido, bem como repercussões de tal estigma na saúde e qualidade de vida dos usuários (Quadro 2).

\section{DISCUSSÃO}

A hanseníase precisa ser vista como uma doença estigmatizante e de grande impacto da condição de vida e saúde dos indivíduos acometidos e de seus familiares. Conforme a literatura, a Índia, o Brasil e a Indonésia respondem por mais de $81 \%$ dos casos da doença em todo o mundo (Tosepu, Gunawan, Effendy, \& Fadmi, 2018). A percepção do estigma entre portadores de hanseníase é referida na literatura como uma condição determinante da redução de qualidade de vida, com repercussões na condição de saúde destes. Embora seja uma doença atualmente tratável com poliquimioterapia persiste o diagnóstico tardio, abandono do tratamento, e ocorrência de casos graves e deformidades (Andrade, et al., 2019). Morgado e col. (Morgado, et al., 2020) em estudo incluindo 180 portadores de hanseníase assistidos em um Centro de Referência do Rio de Janeiro - RJ/Brasil constataram que apenas 4,9\% da amostra sabia a forma correta de transmissão da doença, sendo que o menor grau de conhecimento se relacionou diretamente com maior estigma e sofrimento psíquico. 


\section{Quadro 1. Distribuição da estratégia SPICE para elegibilidade dos estudos}

\begin{tabular}{lll}
\hline & Inclusão & Exclusão \\
\hline S Setting (Cenário) & Diagnóstico confirmado de hanseníase & $\begin{array}{l}\text { Pacientes portadores de outras doenças } \\
\text { infectocontagiosas }\end{array}$ \\
P Perspective (Perspectiva) & Autopercepção de estigma/ preconceito & \\
I Intervention (Intervenção) & A exclusão, estigma e/ou preconceito & \\
C Comparison (Comparação) & Relação entre estigma sofrido/e ou percebido em contextos & \\
& socioeconômicos, étnicos, e níveis de escolaridade & Repercussões do estigma \\
E Evaluation (Avaliação) & Rejo & \\
\hline
\end{tabular}

Fonte:Autoria própria (2021).

Quadro 2. Síntese dos dados coletados

\begin{tabular}{|c|c|c|c|}
\hline Identificação do Estudo & Estigma & Contexto & Repercussões \\
\hline Morgado e col. (2020) & $\begin{array}{l}\text { O maior estigma e preconceito esteve } \\
\text { associado à conceitos errôneos sobre } \\
\text { a doença, baixa renda e menor nível } \\
\text { de escolaridade. }\end{array}$ & $\begin{array}{l}180 \text { usuários assistidos por } \\
\text { um Centro de Referência em } \\
\text { Hanseníase do Rio de Janeiro } \\
\text { - RJ/ Brasil. }\end{array}$ & $\begin{array}{l}\text { Redução da autoestima, depressão } \\
\text { exclusão social. }\end{array}$ \\
\hline Abdelae col. (2020) & $\begin{array}{l}\text { Maior percepção de estigma e } \\
\text { isolamento social foi associado à } \\
\text { menor nível de escolaridade, idade } \\
\text { avançada e ser solteiro. }\end{array}$ & $\begin{array}{l}305 \text { pacientes com } \\
\text { hanseníase assistidos em } \\
\text { base hospitalar da Etiópia }\end{array}$ & $\begin{array}{l}\text { Limitação de atividades }(71,8 \%) \text {, } \\
\text { exclusão social }(55,1 \%) .\end{array}$ \\
\hline $\begin{array}{l}\text { Van'tNoordendee } \\
(2020)\end{array}$ & $\begin{array}{l}\text { Exclusão e preconceito contra } \\
\text { paciente e seus familiares, associado } \\
\text { à presença de filhos, ou maior } \\
\text { presença de deficiência física. }\end{array}$ & $\begin{array}{l}86 \text { indivíduos com } \\
\text { hanseníase e seus familiares } \\
\text { em região Noroeste da } \\
\text { Etiópia. }\end{array}$ & $\begin{array}{l}\text { Sofrimento mental, exclusão social, } \\
\text { estresse e sofrecarga física e psíquica aos } \\
\text { familiares, divórcio e dificuldades em } \\
\text { estabelecer relacionamentos conjugais. }\end{array}$ \\
\hline Jung e col. (2020) & $\begin{array}{l}\text { O diagnóstico de hanseníase é } \\
\text { associado à isolamento social, e } \\
\text { exclusão. Iniciativas de inclusão, e } \\
\text { formação de redes de ajuda auxiliam } \\
\text { em maior dignidade e inclusão. }\end{array}$ & $\begin{array}{l}\text { Estudo envolvendo } 11 \\
\text { portadores de hanseníase e } \\
17 \text { profissionais de saúde } \\
\text { atuantes em hospital da } \\
\text { Índia. }\end{array}$ & $\begin{array}{l}\text { Redução da autoestima, exclusão social, } \\
\text { desemprego e pobreza foram algumas } \\
\text { repercussões da hanseníase relatadas. }\end{array}$ \\
\hline Finottie col. (2020) & $\begin{array}{l}\text { O estigma e a presença de transtornos } \\
\text { mentais comuns tiveram associação } \\
\text { direta com sexo feminino, baixa } \\
\text { renda, faixa etária produtiva e } \\
\text { qualidade de vida insatisfatória. }\end{array}$ & $\begin{array}{lr}206 \quad \text { indivíduos } & \text { com } \\
\text { hanseníase em } & \text { Hospital } \\
\text { Universitário do } & \text { Mato } \\
\text { Grosso, Brasil. } & \end{array}$ & $\begin{array}{l}\text { Verificou-se transtornos mentais comuns } \\
\text { (TMCs) em } 70,4 \% \text { da amostra, estando } \\
\text { tais transtornos diretamente relacionados } \\
\text { ao estigma e sofrimento percebido. }\end{array}$ \\
\hline Cavalcante e col. (2020) & $\begin{array}{l}\text { O estigma esteve associado à } \\
\text { historicidade da hanseníase, sendo } \\
\text { que os próprios indivíduos } \\
\text { acometidos apresentam preconceitos. } \\
\text { Baixo conhecimento sobre doença } \\
\text { relacionou-se à pior estigma }\end{array}$ & 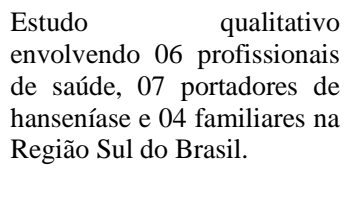 & $\begin{array}{l}\text { O estigma e medos vivenciados atrasam } \\
\text { procura por tratamento, e estão } \\
\text { associados à exclusão e perpetuação do } \\
\text { ciclo de pobreza. }\end{array}$ \\
\hline Palmeira e col.(2020) & $\begin{array}{l}\text { O estigma foi percebido através de } \\
\text { situações excludentes, dentro e fora } \\
\text { do ambiente familiar. O menor } \\
\text { conhecimento sobre a doença, } \\
\text { presença de lesões, e menor } \\
\text { funcionalidade se relacionaram com } \\
\text { maior preconceito. }\end{array}$ & $\begin{array}{l}\text { Estudo com } 17 \text { pacientes } \\
\text { inscritos no Programa } \\
\text { Nacional de Controle da } \\
\text { Hanseníase }(\mathrm{PNCH}) \text { em uma } \\
\text { Unidade Básica de Saúde em } \\
\text { Belém-Pará }\end{array}$ & $\begin{array}{l}\text { O estigma e exclusão reduz a capacidade } \\
\text { de autocuidado, e promove redução da } \\
\text { qualidade de vida e funcionalidade entre } \\
\text { os indivíduos. }\end{array}$ \\
\hline Nascimento e col. (2020) & $\begin{array}{l}\text { O maior estigma e exclusão esteve } \\
\text { relacionado à gravidade da doença, } \\
\text { avanço da idade e vulnerabilidade } \\
\text { social. }\end{array}$ & $\begin{array}{l}\text { Estudo com } 263 \text { indivíduos } \\
\text { portadores de hanseníase. }\end{array}$ & $\begin{array}{l}\text { O diagnóstico de hanseníase e seu } \\
\text { estigma promoveu exclusão social, e } \\
\text { grande impacto psíquico. }\end{array}$ \\
\hline Neiva e Grisotti (2012) & $\begin{array}{l}\text { O estigma esteve associado a } \\
\text { presença de manchas visíveis, menor } \\
\text { conhecimento da doença, e crenças } \\
\text { religiosas. A exclusão se deu no } \\
\text { ambiente social comunitário e } \\
\text { intrafamiliar. }\end{array}$ & $\begin{array}{l}\text { Estudo qualitativo com } 8 \\
\text { mulheres portadoras de } \\
\text { hanseníase, residentes em } \\
\text { comunidade do Vale do } \\
\text { Jequitinhonha, Brasil. }\end{array}$ & $\begin{array}{l}\text { O diagnóstico da doença e estigma } \\
\text { sofrido compromete adesão ao } \\
\text { tratamento, autocuidado, e promove } \\
\text { exclusão social e intrafamiliar. O medo, } \\
\text { e tristeza permeiam a vida das mulheres } \\
\text { acometidas. }\end{array}$ \\
\hline Marahattae col. (2012) & $\begin{array}{l}\text { A presença de deformidades foi o } \\
\text { principal fator associado ao estigma } \\
\text { sofrido. O estigma esteve mais } \\
\text { presente em situações como visitas } \\
\text { aos pacientes, compra de alimentos, } \\
\text { ou mesmo, estabelecimento de } \\
\text { relacionamentos. }\end{array}$ & $\begin{array}{l}\text { Estudo qualitativo com } \\
\text { grupos focais, incluindo } 43 \\
\text { indivíduos com relação } \\
\text { direta com portadores de } \\
\text { hanseníase residentes no } \\
\text { Distrito de Lalitpur, Nepal. }\end{array}$ & $\begin{array}{l}\text { O estigma afeta fatores como interação } \\
\text { social, emprego e casamento dos } \\
\text { indivíduos, comprometendo qualidade de } \\
\text { vida destes de forma significativa. }\end{array}$ \\
\hline Govindharaje col. (2018) & $\begin{array}{l}\text { O medo da doença foi referido por } \\
69 \% \text { dos participantes, sendo que o } \\
\text { maior estigma foi percebido por } \\
\text { mulheres }\end{array}$ & $\begin{array}{l}\text { Estudo realizado na Índia, } \\
\text { envolvendo } 358 \text { indivíduos } \\
\text { afetados pela hanseníase. }\end{array}$ & $\begin{array}{l}\text { O medo da doença promoveu } \\
\text { adoecimento mental, e sofrimento aos } \\
\text { indivíduos. O estigma internalizado ou } \\
\text { percebido pela ocultação do diagnóstico, } \\
\text { e compromete procura por tratamento. }\end{array}$ \\
\hline
\end{tabular}

Fonte:Autoria própria (2021). 


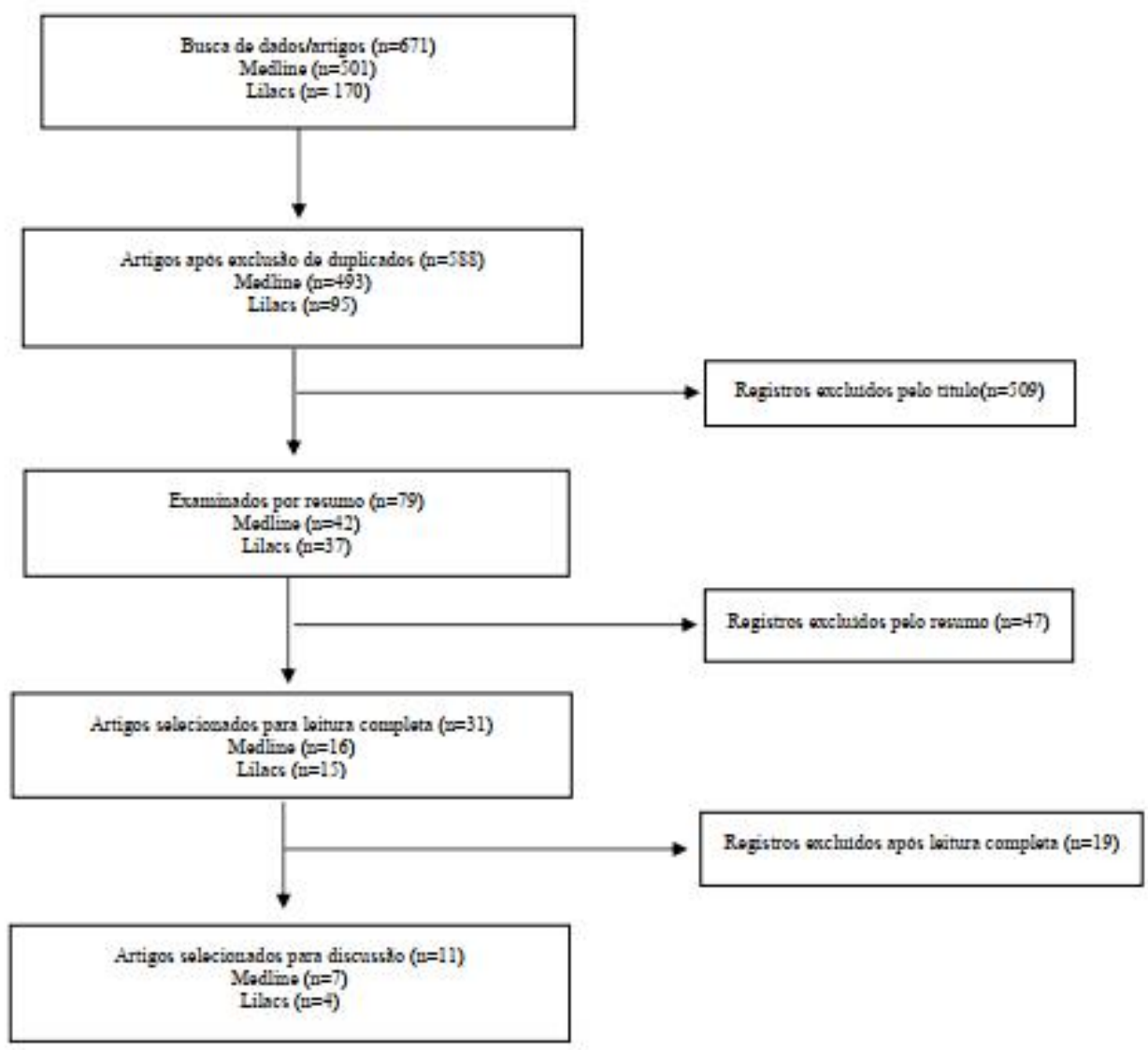

Fonte:Autoria própria (2021).

Fluxograma 1. Triagem e seleção de estudos conforme metodologia PRISMA.

Além de constatarem a adequabilidade da escala os dados apresentados com a utilização da mesma reforçam o estigma percebido/sofrido pelos indivíduos hansênicos. Verificou-se que apenas $4,9 \%$ da amostra sabia a forma correta de transmissão da doença, sendo que o menor grau de conhecimento se relacionou diretamente com maior estigma e sofrimento psíquico. Dentre os sentimentos relatados com o diagnóstico destacaram o medo, tristeza, vergonha, isolamento e depressão. Por seu aspecto histórico e por ser uma doença capaz de causar alterações físicas, que se manifestam por lesões cutâneo mucosas, o simples diagnóstico da doença, mesmo que feito em tempo oportuno para ausência de sequelas gera em suas portadores medos e anseios, além de atitudes excludentes de seus familiares e sociedade. Em estudo realizado em Belém do Pará o maior avanço da doença, sequelas, e redução da funcionalidade foram associados à maior estigma sofrido/percebido (Palmeira, 2020). Em estudo realizado na Etiópia, com 305 portadores de hanseníase os pesquisadores apontam que a deficiência relacionada à hanseníase é um fator contributivo para maior isolamento e/ou exclusão social. Foi utilizada no estudo a Escala de participação e limitação de atividades (SALSA). A maioria dos pacientes $(219,71,8 \%)$ tinha limitação de atividade, sendo que $13,4 \%$ da amostra possuía limitações graves e $8,2 \%$ limitações extremas. Quanto à percepção do estigma, verificouse restrição de participação social em $55,1 \%$ dos indivíduos. A exclusão, e maior sofrimento psíquico advindo da hanseníase esteve associada à baixa escolaridade, idade avançada, e ser solteiro (Abdela, 2020). O instrumento SALSA também foi utilizado no estudo de Nascimento e col (Nascimento et al., 2020). Realizado no município de Picos, Piauí/Brasil. Dentre os 263 indivíduos com hanseníase participantes do estudo verificou-se uma limitação de atividade associada à estágios avançados da doença, bem como correlação direta entre maior idade, menor escolaridade, e menor conhecimento da doença com a percepção de estigma. Contudo, os pesquisadores apontam que mesmo sem lesões visíveis, a própria história cultural da doença pode levar à exclusão, impactando a
O impacto estigmatizante da hanseníase não interfere apenas no indivíduo diagnosticado, mas também em seu círculo familiar e social. A partir da abordagem qualitativa com 86 indivíduos e seus núcleos familiares percebeu-se que a marginalização, restrições sociais, e redução da produtividade são comuns entre tais indivíduos e seus familiares. A discriminação nas comunidades foi referida com grande tristeza pelos participantes, sendo estendida aos familiares dos mesmos. Outro ponto ressaltado foi a ocorrência de divórcio e dificuldades em estabelecer relacionamento conjugal após o diagnóstico de hanseníase. Por outro lado, para os familiares, a presença de um indivíduo hansênicos na família gerava estresse, e muitas vezes maior dispêndio econômico, ocasionando carga de trabalho adicional, abandono escolar e sobrecarga física e emocional (Vant't Noordende, 2020). O sentimento de tristeza, medo da doença, e impacto pela história desta permearam os relatos apresentados em estudo do Vale do Jequitinhonha, Brasil. As oito mulheres entrevistadas relataram exclusão na comunidade e também no ambiente intrafamiliar. No momento do diagnóstico experimentaram insegurança, e receio pela reação que a comunidade poderia apresentar. O medo da doença fez com que houvesse negação do diagnóstico, e retardo no início do tratamento. A negação também foi vista como válvula de escape frente à condição, e esteve relacionada à maior religiosidade, representando a intrínseca relação entre a "lepra" descrita em estudos bíblicos, e o medo de ser excluído no presente pela doença. A omissão do diagnóstico para familiares e amigos também foi atitude comum, ocasionada pelo medo da exclusão. Tais comportamentos geram redução da qualidade de vida, e intenso sofrimento psíquico, que compromete ainda mais a condição de saúde das participantes (Neiva, 2012). O medo da doença também foi referido por $78,5 \%$ dos participantes do estudo realizado por Govindharaje col (Govindharaj, 2018). De um total de 358 participantes, $58 \%$ apresentaram ansiedade, $46 \%$ tristeza e $22 \%$ referiram constrangimento frente ao diagnóstico. A negação e a culpa foi evidenciado em $2 \%$ e $8 \%$ da amostra respectivamente. O estigma foi mais evidente entre indivíduos com deformidades visíveis, 
havendo em todos os indivíduos participantes relato de perda da autoestima, dignidade, e exclusão frente à situações vexatórias e de discriminação. Os autores ressaltam que o estigma pode se apresentar de forma internalizada, percebida ou experimentada. Frente à percepção de maior gravidade da doença os participantes enfrentaram maior estigma internalizado. Já em relação ao estigma experimentado ou percebido muitos usuários apontam a negação ou ocultação do diagnóstico como estratégia de fuga (Govindharaj, 2018). Dentre as consequência do diagnóstico de hanseníase e do estigma sofrido/percebido por seus portadores constatou-se a maior ocorrência de transtornos mentais comuns (TMCs), sobretudo em indivíduos do sexo feminino, baixa renda, e faixa etária produzida. O diagnóstico, associado à exclusão social, repercutiram negativamente na qualidade de vida, culminando com o adoecimento psíquico e maior limitação funcional dos indivíduos. No estudo envolvendo 206 portadores de hanseníase a presença de TMCs foi identificada em 70,4\% da amostra, e a faixa etária de 26-45 anos foi a mais acometida por tais transtornos (Finotti, 2020). Estudo abordando as múltiplas dimensões da Gestão do Cuidado em pacientes hansênicos envolveu pacientes, familiares e profissionais, sendo realizado em um serviço de referência na Região Sul do Brasil. $O$ fato de a hanseníase comprometer a funcionalidade dos indivíduos afetados, bem como, ter um aspecto histórico marcado por relatos desastrosos contribui para perpetuação do estigma associado à doença. $\mathrm{O}$ conhecimento limitado sobre a doença, e os medos, preconceito e anseios dos indivíduos acabam retardando a procura por tratamento, ou determinando a descontinuidade do mesmo. O sofrimento psíquico, exclusão social, e perpetuação do ciclo de pobreza são alguma das consequência da doença na visão de profissionais, portadores e familiares (Cavalcante, 2020).

No estudo realizado por Marahattae col. (Marahatta, et al., 2018) incluindo 43 participantes todos referiram já ter sofrido alguma situação vexatória ou exclusão pelo diagnóstico de hanseníase. Contudo, os participantes relataram que o estigma percebido em relação à doença vem diminuindo ao longo dos anos. $\mathrm{O}$ diagnóstico da doença promove impacto psíquico, sendo que a presença de deformidades e deficiências parece determinante na relação da sociedade com os indivíduos acometidos. $\mathrm{O}$ desconhecimento sobre a doença, e a persistência de tabus sobre a mesma determinam maior exclusão e também ocultação do diagnóstico por muitos participantes. Verificou-se ainda que situações corriqueiras como compra de alimentos, emprego, e visitas à casas de pessoas afetadas pela hanseníase ainda são extremamente comprometidas. Em relação ao casamento, e discriminação por familiares tais traços ainda estão bastante presentes, reforçando a exclusão intrafamiliar. Frente à condição estigmatizante da hanseníase o estudo publicado por Jung e col. (Jung, 2020) retrata uma cooperativa comunitária estruturada na Índia, como forma de inclusão social e combate à hanseníase e suas consequências. No estudo, com abordagem qualitativa, foram realizadas 28 entrevistas envolvendo portadores de hanseníase e funcionários de uma hospital indiano. Os autores apontam que a principal causa para o engajamento dos pacientes na cooperativa era o forte desejo de dignidade e redução do isolamento social. Enfatizouse a importância de um cuidado integral incluindo atendimento holístico, apoio psicossocial, educacional, e inclusão dos núcleos familiares nas abordagens. O estigma e preconceito, associado à exclusão social foi referido por todos os pacientes.

\section{CONSIDERAÇÕES FINAIS}

Todos os estudos apresentados referiram grande estigma, preconceito e exclusão social vivenciada, percebida e/ou internalizada pelos portadores de hanseníase. O desconhecimento da doença, aspectos religiosos, bem como nível de escolaridade e estrato social foram alguns dos aspectos associados à maior estigma. A presença de deformidades, ou manchas visíveis também foram desencadeadoras de maior preconceito, bem como apontadas como motivos de reclusão, e negação do diagnóstico. Dentre as estratégias de enfrentamento de tal estigma foram elencadas a negação, procura de auxílio psíquico, bem como organização de rede ou cooperativas de apoio. A maior ocorrência de depressão, e transtornos mentais comuns também foram dados apresentados na literatura pesquisada.

\section{REFERÊNCIAS}

Abdela SG, Henten Sv, Abegaz SH, Bayuh FB, Zewdu FT, Berhe FT, et al., 2020. Activity limitation and social participation restriction among leprosy patients in Boru Meda Hospital, Amhara Region, Ethiopia. PLoS Negl Trop Dis. 14(09).

Andrade KVFd, Nery JS, Pescarini JM, Ramond A, Santos CAdST, Ichihara MY, et al. 2019. Geographic and socioeconomic factors associated with leprosy treatment default: An analysis from the 100 Million Brazilian Cohort. PLoS Negl Trop Dis., 13(9).

Araújo BG, Brito DPC, Coelho VAT, Nascimento EdS, Pereira MCS, Lacerda LG, et al., 2019. Perfil sociodemográfico e epidemiológico de novos casos de hanseníase no município de Almenara - MG. Id on Line - Revista Multidisciplinar e de Psicologia. Outubro: p. 410423.

Araújo DAL, Brito KKG, Santana MF, Soares VL, Soares MJGO. 2016. Caracterização da qualidade de vida de pessoas com hanseníase em tratamento ambulatorial. Revista de Pesquisa: Cuidado é Fundamental Online. Nov: p. 5010-5016.

Brasil. 2020. Boletim Epidemiológico especial: Hanseníase. Boletim Epidemiológico. Brasília: Secretaria de Vigilância em Saúde, Ministério da Saúde. Report No.: 1.

Carvalho APVd, Silva V, Grande AJ. 2013. Avaliação do risco de viés de ensaios clínicos randomizados pela ferramenta da colaboração Cochrane. Diagn Tratamento. 18(1): p. 38-44.

Cavalcante MDMA, Larocca LM, Chaves MMN. 2020. Múltiplas dimensões da gestão do cuidado à hanseníase e os desafios para a eliminação. Rev. esc. enferm. USP. 54.

Finotti RdFC, Andrade ACdS, Souza DPOd. 2020. Transtornos mentais comuns e fatores associados entre pessoas com hanseníase: análise transversal em Cuiabá, 2018. Epidemiol. Serv. Saúde. 29(4).

Govindharaj P, Srinivasan S, Darlong J. 2018. Perception toward the Disease of the People Affected by Leprosy. International Journal of Mycobacteriology. 7(3).

Jung SH, Han HW, Koh H, Yu SY, Nawa N, Morita A, et al. 2020. Patients help other patients: Qualitative study on a longstanding community cooperative to tackle leprosy in India. PLos Negl Trop Dis., 14(1).

Liberati A, Altman DG, Tetzlaff J, Mulrow C, Gotzsche PC, Ioannidis JPA, et al. 2009. The PRISMA statement for reporting systematic reviews and meta-analyses of studies that evaluate healthcare interventions: explanation and elaboration. BMJ. 339(1).

Lopes EFB, Silva LSAd, Rotta dS, Oliveira JHMD, Menezes Rd, Nakamura L, et al. Educação em saúde: uma troca de saberes no combate ao estigma da hanseníase. Braz. J. of Develop. 2020 Fevereiro: p. 5350-5368.

Marahatta SB, Amatya R, Adhikari S, Giri D, Lama S, Kaehler N, et al., 2018. Perceived stigma of leprosy among community members and health care providers in Lalitpur district of Nepal: A qualitative study. PLoS Negl Trop Dis., 13(12).

Morgado FFdR, Silveira EMKd, Nascimento LPRd, Sales AM, Nery JAdC, Sarno EN, et al. Psychometric assessment of the EMIC Stigma Scale for Brazilians affected by leprosy. Plos One. 2020; 15(9).

Nascimento DdS, Ramos Júnior AN, Araújo OD, Macêdo SFd, Silva GVd, Lopes WMPS, et al., 2020. Limitação de atividade e restrição à participação social em pessoas com hanseníase: análise transversal da magnitude e fatores associados em município hiperendêmico do Piauí, 2001 a 201. Epidemiol. Serv. Saúde., 29(3).

Neiva RJ, Grisotti M. 2012. Representações do estigma da hanseníase nas mulheres do Vale do Jequitinhonha-MG. Physis. 29(1).

Palmeira IP, Moura JN, Epifane SG, Ferreira AMR, Boulhosa MF. 2020. Percepção de pacientes com hanseníase sobre suas necessidades humanas básicas alteradas: indícios para o autocuidado. Rev. Pesqui. (Univ. Fed. Estado Rio J., Online).; 12: p. 324-329. 
Pinheiro MG, Miranda FANd, Simpson A, Carvalho FPBd, Ataide CA, Lira ALBdC. 2018. Compreendendo a "alta em hanseníase": uma análise de conceito. Rev. Gaúcha Enferm. junho: p. e63290.

Sousa AAd, Oliveira FJFd, Costa ACPdJ, Santos Neto M, Cavalcante EFdO, Ferreira GN. 2013. Adesão ao tratamento da hanseníase por pacientes acompanhados em Unidades Básicas de Saúde de Imperatriz - MA. Sanare - Revista de Políticas Públicas. p. 6-12.
Tosepu R, Gunawan J, Effendy DS, Fadmi FR. 2018. Stigma and increase of leprosy cases in SouthEast Sulawesi Province, Indonesia. African Health Sciences., 18(1): p. 29-31.

Vant't Noordende AT, Aycheh MW, Schippers A. 2020. The impact of leprosy, podoconiosis and lymphatic filariasis on family quality of life: A qualitative study in Northwest Ethiopia. PLoS Negl Trop Dis., 14(03). 